\title{
On the role of heterogeneity in concentrated solid- solution alloys in enhancing their irradiation resistance
}

\author{
Shijun Zhao ${ }^{1, a)}$ \\ ${ }^{1}$ Department of Mechanical Engineering, City University of Hong Kong, Hong Kong, China, 999077; and Shenzhen Research Institute of City University \\ of Hong Kong, Shenzhen, China, 518057

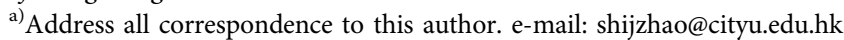 \\ This paper has been selected as an Invited Feature Paper. \\ Received: 15 September 2019; accepted: 14 October 2019
}

Concentrated solid-solution alloys (CSAs) demonstrate excellent mechanical properties and promising irradiation resistance depending on their compositions. Existing experimental and simulation results indicate that their heterogeneous structures induced by the random arrangement of different elements are one of the most important reasons responsible for their outstanding properties. Nevertheless, the details of this heterogeneity remain unclear. Specifically, which properties induced by heterogeneity are most relevant to their irradiation response? In this work, we scrutinize the role of heterogeneity in CSAs played in damage evolution in different aspects through atomistic simulations, including lattice misfit, thermodynamic mixing, point defect energetics, point defect diffusion, and dislocation properties. Our results reveal that structural parameters, such as lattice misfit and enthalpy of mixing, are generally not suitable to assess their irradiation response under cascade conditions. Instead, atomic-level defect properties are the keys to understand defect evolution in CSAs. Therefore, tuning chemical disorder to tailor defect properties is a possible way to further improve the irradiation performance of CSAs.

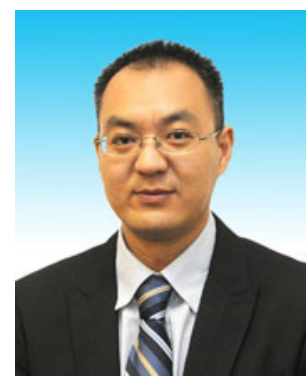

Shijun Zhao

Dr. Shijun Zhao is an Assistant Professor at City University of Hong Kong. Dr. Zhao received his Bachelor's degree in Physics in 2008 and his Ph.D. degree in Nuclear Engineering in 2013, both from Peking University. His Ph.D. research centered on the radiation effects in ceramics and low-dimensional materials based on ab initio methods and molecular dynamics simulations. After graduation, Dr. Zhao conducted his postdoctoral research at the College of Engineering at Peking University for two years, with a focus on excited electron dynamics in materials under high-energy ion or laser irradiation using ab initio methods. Prior to joining City University of Hong Kong in 2018, he was a postdoctoral research associate at Oak Ridge National Laboratory, working on the irradiation effects in concentrated solid-solution alloys. Dr. Zhao's current research group works on computational defect properties. Specifically, his group aims to understand defect thermodynamics, defect production, defect migration, and defect evolution in different materials under deformation or irradiation conditions. The defects considered include point defects, impurities, dislocations, stacking faults, grain boundaries, and surfaces, etc. The materials concerned including nuclear structural materials and nuclear fuels span from metallic alloys to ceramics. For this purpose, different simulation techniques at different scales are concurrently or sequentially employed. Dr. Zhao has authored and co-authored more than 50 technical articles with more than 1400 citations in the field of defect properties in materials.

\section{Introduction}

Concentrated solid-solution alloys (CSAs), such as mediumentropy alloys (MEAs) and high-entropy alloys (HEAs), are composed of multiple principal elements all at high concentrations $[1,2,3]$. With different high-concentration elements randomly arranged in a simple lattice, CSAs are characterized by their inherent chemical disorder, resulting in atomic-level heterogeneity that significantly influences their properties [4]. In fact, it has been proved that both the excellent mechanical properties and irradiation resistance in CSAs relate to their heterogeneous structures. For example, strengthening of CSAs can be predicted by considering dislocation interactions with 
random local concentration fluctuations due to heterogeneity [5]. The radiation resistance of CSAs can also find its origin in their heterogeneous structures, which lead to heterogeneity of defect diffusion [4] and dislocation movement [6, 7, 8]. Nevertheless, the detailed role of heterogeneity played in the damage evolution is still elusive. Specifically, what kinds of properties that are most influenced by the heterogeneous structures contribute predominantly to their irradiation tolerance?

In CSAs, every atom is surrounded by unique local atomic environments, which lead to spatially different local propertiesheterogeneity depending on alloying compositions. Experiments have demonstrated that CSAs with different compositions exhibit distinct damage tolerance. For example, less structural damage is detected in $\mathrm{NiCo}$ than in $\mathrm{NiFe}$ under the same radiation conditions based on the Rutherford backscattering spectrometry measurement [9]. The same technique also reveals that irradiated $\mathrm{NiCoCr}$ exhibits less defect than NiFe [8]. These results indicate that by tuning alloying composition and concentration, the degree of heterogeneity can be modified and a better irradiation performance may be achieved. Different criteria have been proposed as a measure of the heterogeneity in affecting irradiation resistance of CSAs, such as the thermodynamic mixing energy [10], migration energy distributions [11], chemically-biased diffusion $[7,12,13]$, and steady dislocation velocities [8]. However, these criteria are proposed based on limited simulation data, either from one set of empirical potential or from limited alloy systems. To evaluate these factors from experiments would be challenging as it is difficult to separate these different effects. A comprehensive understanding of these effects induced by the heterogeneous structures of CSAs is highly desirable to gain a more fundamental basis to guide alloy design with improved irradiation resistance.

In this article, we assess the effects of structural heterogeneity on different properties of CSAs and how these properties correlate with the irradiation tolerance of CSAs. Two independently developed embedded-atom method (EAM) potentials are employed. Based on the available experimental results, several alloy systems described by these two potentials are investigated, including $\mathrm{Ni}_{0.5} \mathrm{Co}_{0.5}, \quad \mathrm{Ni}_{0.5} \mathrm{Fe}_{0.5}, \quad \mathrm{Ni}_{0.8} \mathrm{Cr}_{0.2}$, $\mathrm{Ni}_{0.4} \mathrm{Fe}_{0.4} \mathrm{Cr}_{0.2}$, and $\mathrm{Ni}_{0.333} \mathrm{Co}_{0.333} \mathrm{Cr}_{0.333}$ (NiCoCr). Different properties of these CSAs, including lattice misfit, enthalpy of mixing, defect migration energy distributions, stacking fault energy distributions, and dislocation motion modes, are calculated and compared with their damage buildup that is simulated by accumulated collision cascade. This work aimed to reveal what kinds of properties that are influenced by heterogeneity play the dominant roles in governing the irradiation resistance of CSAs. The results are expected to gain an overview of the most relevant quantities among those various parameters previously reported.

\section{Result}

To validate the potentials used in this study, we first calculated the lattice parameters of all considered alloy systems. The results are summarized in Table I. The obtained lattice parameters for pure metals are consistent with those in the original reports of potential developers [14, 15]. For disordered alloys, the lattice parameters were determined by fitting the energy-volume relations of a 4000-atoms supercell with randomly distributed elements. In this work, we employed the random function in python to shuffle the index of 4000 atoms. By generating thousands of these random structures, the random distribution of elements is ensured. In general, all three potentials predict a larger lattice constant in disordered fcc alloys than that of fcc pure $\mathrm{Ni}$. With the calculated lattice parameters, we have studied different properties of these CSAs.

\section{Lattice misfit}

Previous studies indicate that the volume misfit to a large extent determines the strength of CSAs [5]. We have calculated the volume misfit parameters according to the following formula [5]:

$$
\delta=\frac{1}{\bar{V}} \sqrt{\sum_{n} c_{n}\left(\Delta V_{n}\right)^{2}},
$$

where $c_{n}$ is the concentration of element $n, \Delta V_{n}$ is the average misfit volume of element $n$, and $\bar{V}$ is the atomic volume of the alloy. In this formula, $\delta$ is the concentration-weighted mean square misfit volume. Following the previously proposed method to calculate the misfit volume in concentrated alloys [16], the $\delta$ parameters are calculated and provided in Table II. Here, we employed a fcc supercell with 864 atoms, and the volume change induced by replacing each atom by another atom with different atomic type was recorded. It can be seen that $\mathrm{Ni}_{0.8} \mathrm{Cr}_{0.2}$ possesses a larger $\delta$ than $\mathrm{Ni}_{0.5} \mathrm{Fe}_{0.5}$ with the Bonny potential even larger than that of $\mathrm{Ni}_{0.4} \mathrm{Fe}_{0.4} \mathrm{Cr}_{0.2}$. Based

TABLE I: Calculated lattice parameters (in $\AA$ ) for all considered alloy systems using different potentials. For hcp $\mathrm{Co}$, values of $a$ and $c$ are provided.

\begin{tabular}{lcc}
\hline \hline & Bonny & Zhou \\
\hline $\mathrm{Ni}(\mathrm{fcc})$ & 3.523 & 3.519 \\
$\mathrm{Fe}(\mathrm{bcc})$ & 2.862 & 2.866 \\
$\mathrm{Fe}(\mathrm{fcc})$ & 3.560 & 3.628 \\
$\mathrm{Cr}(\mathrm{bcc})$ & 2.882 & 2.855 \\
$\mathrm{Cr}(\mathrm{fcc})$ & 3.534 & 3.655 \\
$\mathrm{Co}(\mathrm{hcp})$ & $\ldots$ & $2.5120 / 4.075$ \\
$\mathrm{Co}(\mathrm{fcc})$ & $\ldots$ & 3.549 \\
$\mathrm{Ni}_{0.5} \mathrm{Co} \mathrm{O}_{0.5}$ & $\ldots$ & 3.5333 \\
$\mathrm{Ni}_{0.5} \mathrm{Fe}_{0.5}$ & 3.5335 & 3.5610 \\
$\mathrm{Ni}_{0.8} \mathrm{Cr}_{0.2}$ & 3.5921 & 3.5279 \\
$\mathrm{Ni}_{0.4} \mathrm{Fe}_{0.4} \mathrm{Cr}_{0.2}$ & 3.5550 & $\ldots$ \\
$\mathrm{Ni}_{0.333} \mathrm{Co}_{0.333} \mathrm{Cr}_{0.333}$ & $\ldots$ & 3.5448 \\
\hline \hline
\end{tabular}


TABLE II: Calculated misfit volume parameters for considered alloys.

\begin{tabular}{lcc}
\hline \hline & Bonny & Zhou \\
\hline $\mathrm{Ni}_{0.5} \mathrm{Co}_{0.5}$ & $\ldots$ & 0.012 \\
$\mathrm{Ni}_{0.5} \mathrm{Fe}_{0.5}$ & 0.010 & 0.043 \\
$\mathrm{Ni}_{0.8} \mathrm{Cr}_{0.2}$ & 0.079 & 0.021 \\
$\mathrm{Ni}_{0.4} \mathrm{Fe}_{0.4} \mathrm{Cr}_{0.2}$ & 0.030 & $\ldots$ \\
$\mathrm{Ni}_{0.333} \mathrm{Co}_{0.333} \mathrm{Cr}_{0.333}$ & $\ldots$ & 0.025 \\
\hline \hline
\end{tabular}

on the Zhou potential, $\mathrm{Ni}_{0.5} \mathrm{Fe}_{0.5}$ possesses the largest $\delta$ parameter, whereas $\mathrm{Ni}_{0.5} \mathrm{Co}_{0.5}$ the smallest. The $\delta$ parameters in $\mathrm{Ni}_{0.8} \mathrm{Cr}_{0.2}$ and $\mathrm{Ni}_{0.333} \mathrm{Co}_{0.333} \mathrm{Cr}_{0.333}$ are quite similar.

\section{Thermodynamic mixing}

The mixing energy of CSAs has been proposed as an indicator of irradiation performance of CSAs. It shows that for $\mathrm{Ni}_{1-x} \mathrm{Fe}_{x}$ alloys, the defect number correlates linearly with the mixing energy of the alloy [10]. Here, we calculate the mixing energies of considered CSAs according to

$$
E_{\mathrm{mix}}=E_{\mathrm{tot}}-\sum_{i} n_{i} \varepsilon_{i}
$$

where $E_{\text {tot }}$ is the total energy of the alloy system, $n_{i}$ is the number of element $i$, and $\varepsilon_{i}$ is its per-atom energy in the bulk pure metal counterpart. The results are summarized in Table III. In calculating $E_{\text {tot }}$, we randomly distributed different elements in a fcc 4000-atoms supercell. The energies of 200 generated configurations were calculated and averaged. It shows that Bonny $\mathrm{Ni}_{0.5} \mathrm{Fe}_{0.5}$ has a lower $E_{\text {mix }}$ than $\mathrm{Ni}_{0.8} \mathrm{Cr}_{0.2}$, whereas $Z_{\text {hou }} \mathrm{Ni}_{0.5} \mathrm{Fe}_{0.5}$ has a higher $E_{\text {mix }}$ than both $\mathrm{Ni}_{0.8} \mathrm{Cr}_{0.2}$ and $\mathrm{Ni}_{0.5} \mathrm{Co}_{0.5}$. The mixing energy for $\mathrm{Ni}_{0.4} \mathrm{Fe}_{0.4} \mathrm{Cr}_{0.2}$ is rather large (and positive) from the Bonny potential, whereas that of $\mathrm{Ni}_{0.333} \mathrm{Co}_{0.333} \mathrm{Cr}_{0.333}$ is only slightly negative from the Zhou potential. Note that the effect of short-range order is not taken into account in our calculations. However, our mixing energy for $\mathrm{Ni}_{0.5} \mathrm{Fe}_{0.5}$ is comparable to the previous result of Jin et al. [10], suggesting limited effects of local ordering on the enthalpy of mixing.

\section{Defect migration energies}

The migration and diffusion of defects are one of the most important processes contributing to defect evolution under irradiation. Because of the atomic-level heterogeneity in CSAs, defect migration energies depend strongly on the local atomic environment. The calculated distributions of migration energies for vacancies and interstitials in considered alloys are provided in Fig. 1. The results from the Bonny potential are presented in the upper half of the figure, whereas those calculated from the Zhou potential are provided in the lower half. These migration barriers were calculated in a $10 \times 10 \times$ 10 supercell containing 4000 atoms with the nudged elastic
TABLE III: Calculated mixing energies for considered alloys (eV/atom).

\begin{tabular}{lcr}
\hline \hline & Bonny & Zhou \\
\hline $\mathrm{Ni}_{0.5} \mathrm{Co}_{0.5}$ & $\ldots$ & 0.005 \\
$\mathrm{Ni}_{0.5} \mathrm{Fe}_{0.5}$ & -0.075 & 0.038 \\
$\mathrm{Ni}_{0.8} \mathrm{Cr}_{0.2}$ & 0.154 & -0.011 \\
$\mathrm{Ni}_{0.4} \mathrm{Fe}_{0.4} \mathrm{Cr}_{0.2}$ & 0.115 & $\ldots$ \\
$\mathrm{Ni}_{0.333} \mathrm{CO}_{0.333} \mathrm{Cr}_{0.333}$ & $\ldots$ & -0.004 \\
\hline \hline
\end{tabular}

band (NEB) [17] method as implemented in the LAMMPS code. The convergence criteria of force were $10^{-6} \mathrm{eV} / \AA$ with 16 intermediate images.

Figure 1 suggests that different interatomic potentials result in distinct distributions of defect migration energies. Generally, the distributions from the Bonny potential are wider than those from the Zhou potential, suggesting a rougher defect energy landscape in the Bonny potential. Based on the results from the Bonny potential, it can be seen that the migration barriers of vacancies shift to the low energy direction, whereas those of interstitials move to the high energy direction, resulting significantly an overlap region between their distributions. Comparing the three alloys considered, the range of the overlap region in $\mathrm{Ni}_{0.5} \mathrm{Fe}_{0.5}$ and $\mathrm{Ni}_{0.8} \mathrm{Cr}_{0.2}$ is similar, whereas $\mathrm{Ni}_{0.4} \mathrm{Fe}_{0.4} \mathrm{Cr}_{0.2}$ shows the largest overlap region among these three alloys.

The results from the Zhou potential show that the migration energies in $\mathrm{Ni}_{0.5} \mathrm{Co}_{0.5}$ show no overlap region, in contrast to the other three alloys. In $\mathrm{Ni}_{0.5} \mathrm{Fe}_{0.5}, \mathrm{Ni}_{0.8} \mathrm{Cr}_{0.2}$ and $\mathrm{Ni}_{0.333} \mathrm{Co}_{0.333} \mathrm{Cr}_{0.333}$, the migration energies of vacancies lowered to around $0.6 \mathrm{eV}$, whereas those of interstitial extended to about $0.5-0.6 \mathrm{eV}$, resulting in a slight overlap region. This overlap region is narrower than results calculated from the Bonny potential, and it will induce different defect accumulation behaviors under accumulated ion irradiations.

\section{Defect diffusion}

Diffusion of point defects at finite temperatures is simulated directly by MD and the diffusion coefficients are calculated. In these simulations, a $10 \times 10 \times 10$ supercell containing 4000 atoms was used. A vacancy or interstitial was introduced into the supercell by removing or adding an atom randomly into the lattice. The simulations were carried out within an NPT ensemble, and the total simulation time was $200 \mathrm{~ns}$. The atomic square displacement (ASD) of all atoms was calculated at different temperatures, and the diffusion coefficients were then obtained from the slope of the time-dependent ASD according to Eisenstein's formula. The results are represented by the Arrhenius plot as shown in Fig. 2. The fitted parameters, $E_{\mathrm{a}}$ and $D_{0}$, from the relation $D^{*}=D_{0} \exp \left(-E_{\mathrm{a}} / k_{\mathrm{B}} T\right)$ are also given.

Based on the Bonny potential, the interstitial diffusion becomes slower, whereas vacancy diffusion becomes faster in 


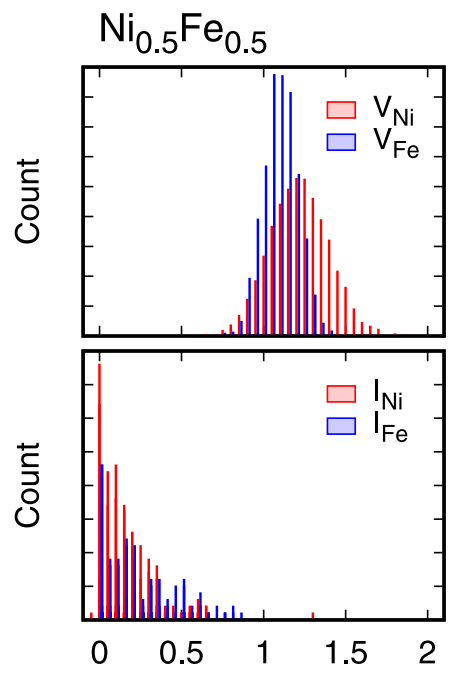

(a) Migraion energy (eV)

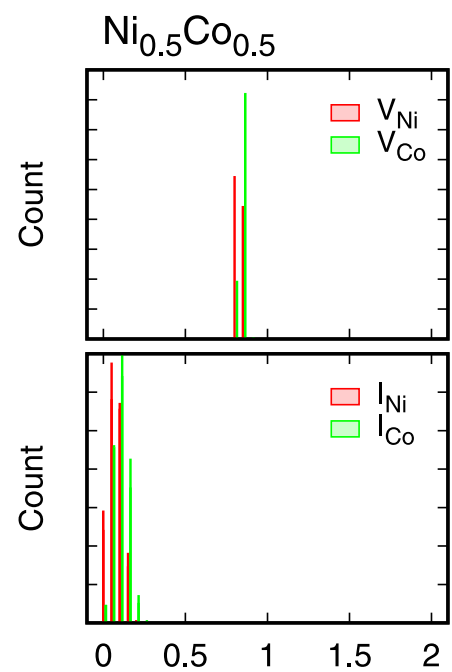

(d) Migraion energy (eV)

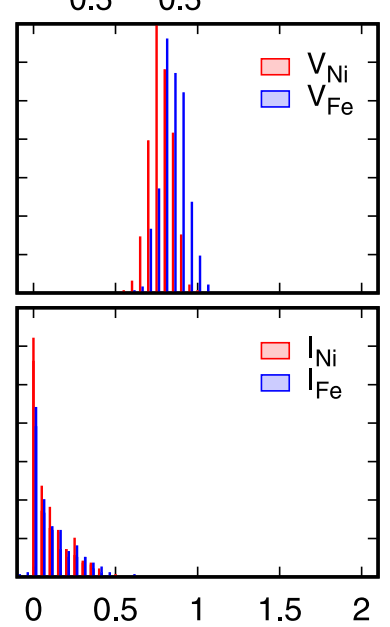

(e) Migraion energy $(\mathrm{eV})$

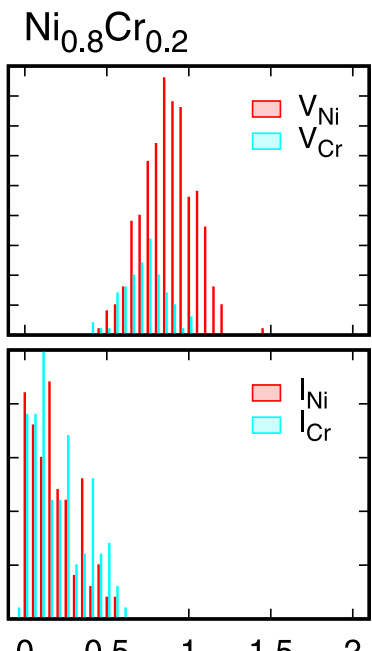

(b) Migration energy (eV)
$\mathrm{Ni}_{0.8} \mathrm{Cr}_{0.2}$

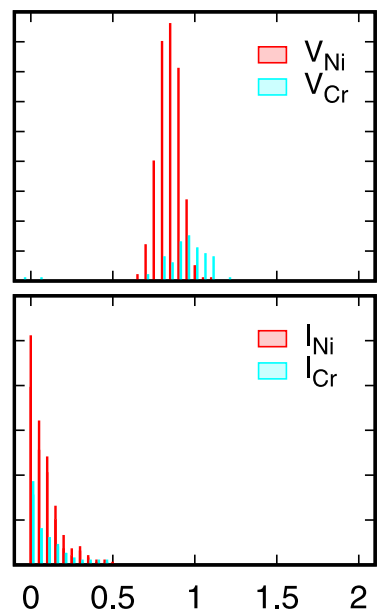

$\mathrm{Ni}_{0.4} \mathrm{Fe}_{0.4} \mathrm{Cr}_{0.2}$
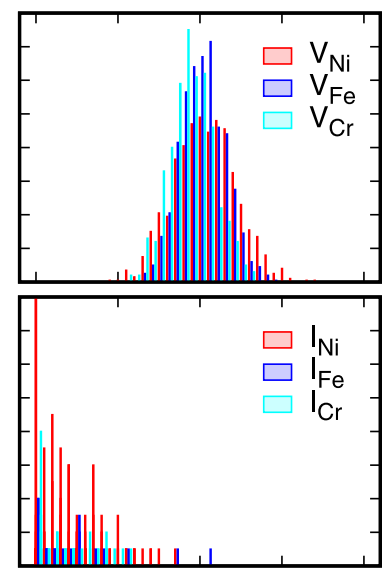

$\begin{array}{lllll}0 & 0.5 & 1 & 1.5 & 2\end{array}$

(c) Migration energy (eV)

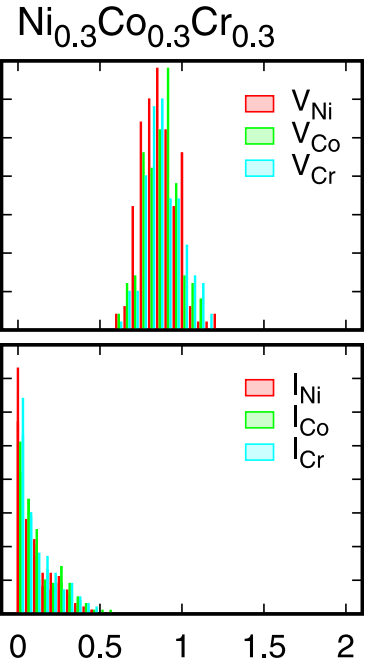

(g) Migration energy (eV)

Figure 1: Calculated defect migration energy distributions in considered alloys. The upper half represents results from the Bonny potential, whereas the results in the lower half are obtained from the Zhou potential.

CSAs than that in pure Ni. Specifically, Fig. 2 suggests interstitial diffusion is the slowest in $\mathrm{Ni}_{0.5} \mathrm{Fe}_{0.5}$ and $\mathrm{Ni}_{0.8} \mathrm{Cr}_{0.2}$, whereas vacancy diffusion is the fastest in $\mathrm{Ni}_{0.4} \mathrm{Fe}_{0.4} \mathrm{Cr}_{0.2}$ and $\mathrm{Ni}_{0.8} \mathrm{Cr}_{0.2}$. The results from the Zhou potential is consistent qualitatively with those from the Bonny potential in terms of interstitial diffusion, which also predicts the slowest diffusion of interstitial atoms in $\mathrm{Ni}_{0.5} \mathrm{Fe}_{0.5}$. Nevertheless, the Zhou potential suggests that vacancy diffusion becomes slower in CSAs instead of faster as observed in the Bonny potential. In particular, $\mathrm{Ni}_{0.333} \mathrm{Co}_{0.333} \mathrm{Cr}_{0.333}$ shows the slowest vacancy diffusion.

One noticeable feature in Fig. 2 is that the activation energies for interstitial diffusion fitted from the Arrhenius relation are different from the averaged migration barriers calculated at $0 \mathrm{~K}$ as presented in Fig. 1, although the general trend is similar, i.e., interstitial diffusion possesses a higher activation (migration) energy. The deviation is more pronounced in CSAs, presumably due to the correlation effects between defect jumps. Besides, larger differences in activation energies between CSAs and $\mathrm{Ni}$ are observed from the Bonny potential than those from the Zhou potential.

\section{Preferential diffusion}

In CSAs, defect diffusion proceeds through a chemically biased mechanism $[7,18]$. The defect tends to preferentially exchange positions with a certain constituent component, leading to a higher diffusion coefficient of the corresponding element. It has been shown that this chemically biased atomic diffusion is the key for understanding defect evolution in CSAs [13]. Here, the preferential diffusion is evaluated by calculating the ratio of 

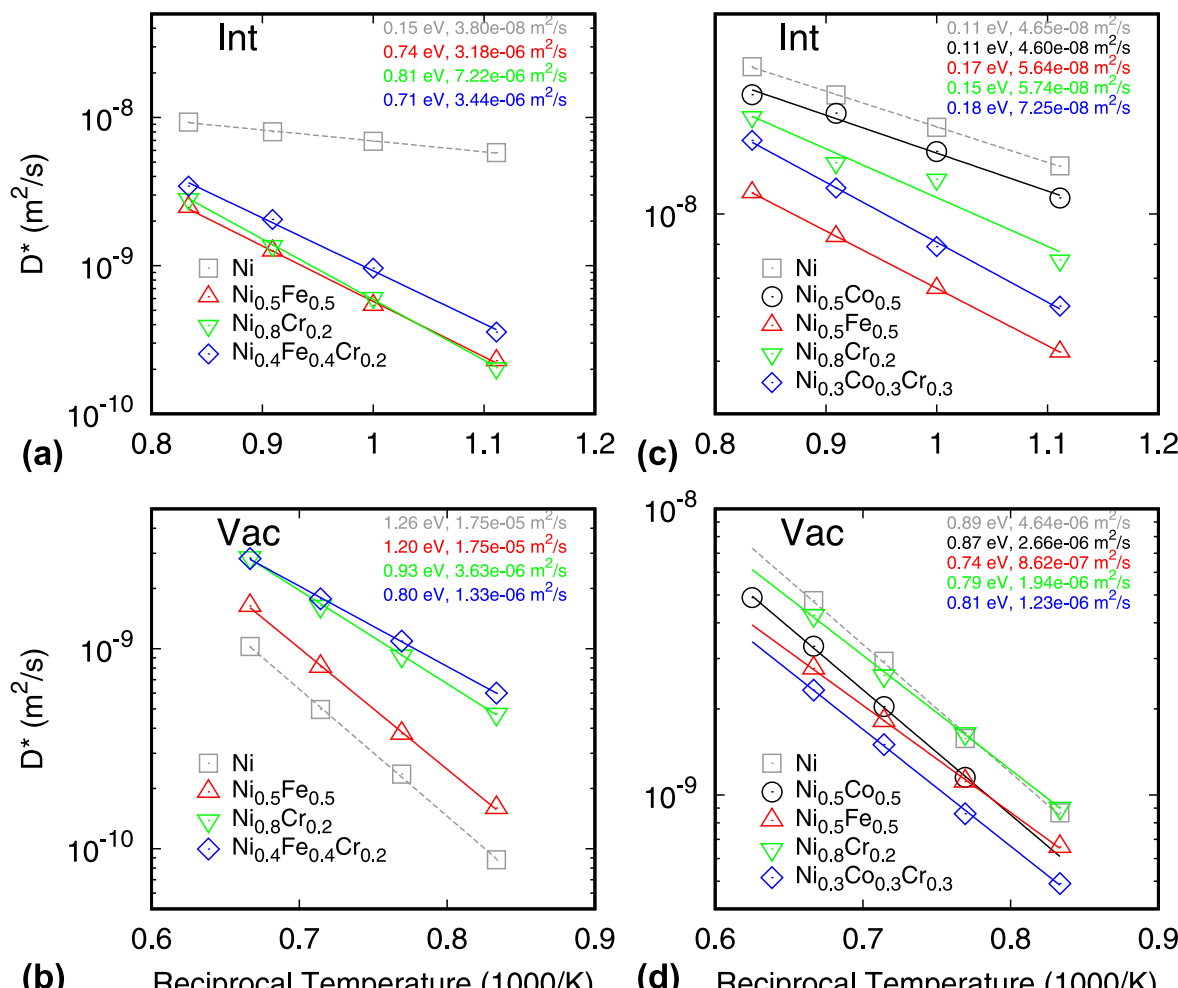

(d) Reciprocal Temperature (1000/K)

Figure 2: Diffusion coefficients of interstitial and vacancy defects in the considered alloys. The results calculated using the Bonny potential are shown in the left column, whereas those from the Zhou potential are shown in the right.

the partial diffusion coefficients in the considered alloys. The results are given in Fig. 3.

In this figure, the ratio is represented by $D_{\mathrm{X}}^{*} / D_{\mathrm{Ni}}^{*}$, where $\mathrm{X}$ denotes the other elements in the alloy. In $\mathrm{Ni}_{0.4} \mathrm{Fe}_{0.4} \mathrm{Cr}_{0.2}$ $\left(\mathrm{Ni}_{0.333} \mathrm{Co}_{0.333} \mathrm{Cr}_{0.333}\right), D_{\mathrm{Fe}(\mathrm{Co})}^{*} / D_{\mathrm{Ni}}^{*}$ is presented by blue lines, whereas $D_{\mathrm{Cr}}^{*} / D_{\mathrm{Ni}}^{*}$ is denoted by pink lines. Based on the results from the Bonny potential, the ratios for interstitials are less than 1, suggesting preferential diffusion of $\mathrm{Ni}$ flux in all alloys. The largest disparity is seen in $D_{\mathrm{Fe}}^{*} / D_{\mathrm{Ni}}^{*}$ in $\mathrm{Ni}_{0.5} \mathrm{Fe}_{0.5}$ and $D_{\mathrm{Fe}}^{*} / D_{\mathrm{Ni}}^{*}$ in $\mathrm{Ni}_{0.4} \mathrm{Fe}_{0.4} \mathrm{Cr}_{0.2}$. For vacancy diffusion, $D_{\mathrm{Fe}}^{*} / D_{\mathrm{Ni}}^{*}$ in $\mathrm{Ni}_{0.4} \mathrm{Fe}_{0.4} \mathrm{Cr}_{0.2}$ is also less than 1. Among these alloys, $\mathrm{Ni}_{0.4} \mathrm{Fe}_{0.4} \mathrm{Cr}_{0.2}$ seems to have the largest diffusion disparity in the partial diffusion coefficient. The results from the Zhou potential indicate that $D_{\mathrm{Cr}}^{*} / D_{\mathrm{Ni}}^{*}$ in $\mathrm{Ni}_{0.333} \mathrm{Co}_{0.333} \mathrm{Cr}_{0.333}$ is the lowest for both interstitial and vacancy diffusion, whereas $D_{\mathrm{Co}}^{*} / D_{\mathrm{Ni}}^{*}$ is the highest, causing the largest disparity in the diffusion coefficients of constituent components. Therefore, for these alloys, the chemically biased diffusion effects are the most pronounced in the ternary systems.

\section{Dislocation properties}

The migration and diffusion of point defects lead to the formation of defect clusters, such as dislocations. In CSAs, the properties of dislocations are related to the distribution of their stacking fault energies (SFEs) [19]. Therefore, SFE distributions in the considered CSAs were first studied and are shown in Fig. 4. In these calculations, the stacking fault size was fixed to $20 \times 40$ along the [112] and [110] directions. At a given box size and alloy composition, a total of 3000 random realizations were performed for each alloy composition. The obtained SFE distribution is fitted to the Gaussian function, and the resulting average value $\mu$ and standard deviation $\sigma$ are provided. From the fitting results, both the Bonny and Zhou potentials predict similar SFEs for $\mathrm{Ni}_{0.5} \mathrm{Fe}_{0.5}$ and $\mathrm{Ni}_{0.8} \mathrm{Cr}_{0.2}$. Nevertheless, the fluctuations in SFE distributions exhibit different features. $\mathrm{Ni}_{0.4} \mathrm{Fe}_{0.4} \mathrm{Cr}_{0.2}$ possesses the largest fluctuations among the three considered CSAs based on the Bonny potential, whereas $\mathrm{Ni}_{0.333} \mathrm{Co}_{0.333} \mathrm{Cr}_{0.333}$ shows the largest fluctuations based on the Zhou potential. Another feature is that the width of SFE distribution is relatively small from the Zhou potential. Besides, the differences among the $\sigma$ values for the considered alloys are small (less than $0.5 \mathrm{~mJ} / \mathrm{m}^{2}$ ).

The velocity of an [110]/2 edge dislocation in the considered alloys is then studied by applying external stresses, as carried out in previous works [20,21]. Briefly, a simulation cell oriented along the [110], [111], and [112] directions was used with the dimensions around $30 \times 24 \times 22 \mathrm{~nm}^{3}$. The edge dislocation was introduced in the center of the cell by adding an extra atomic layer into the upper half of the crystal, with the 

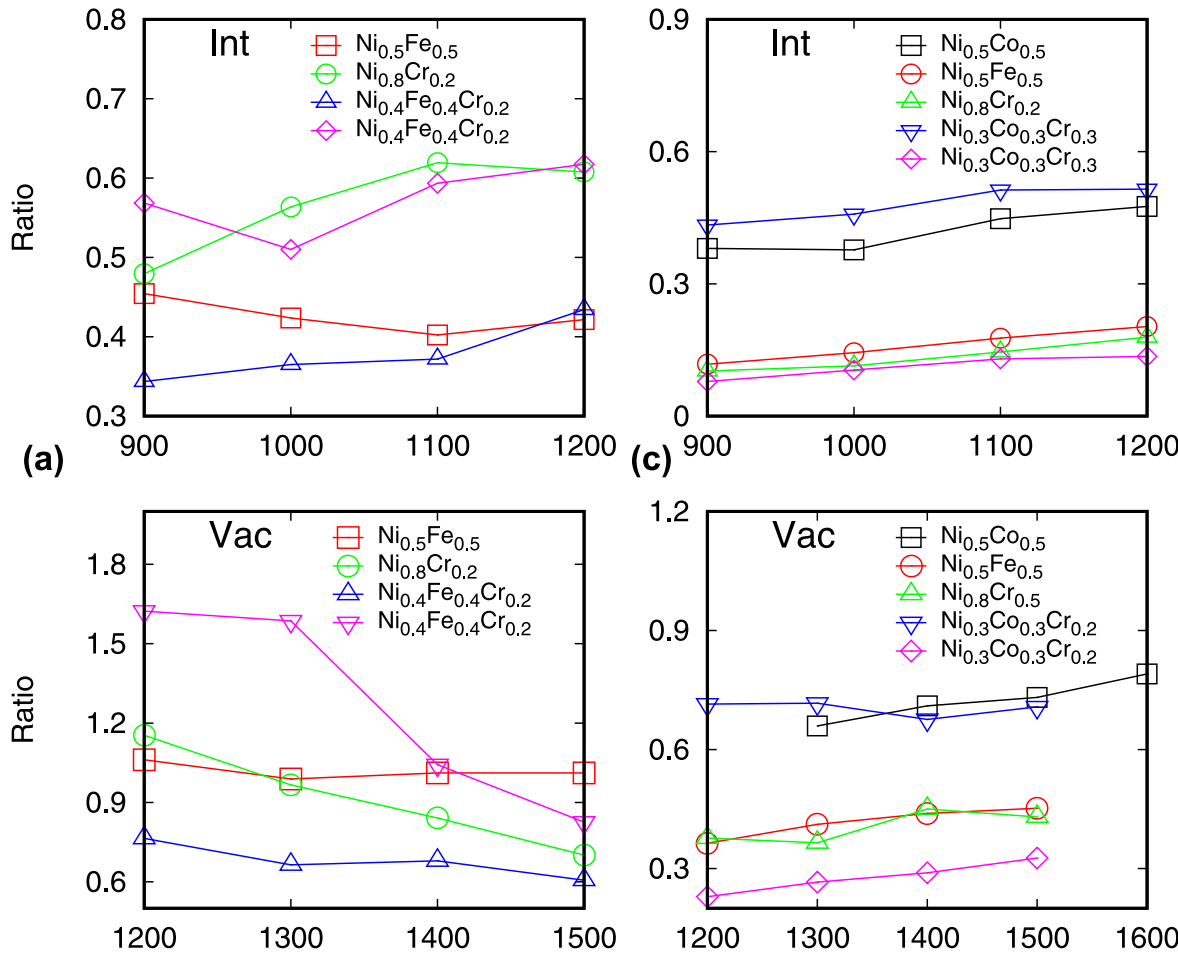

(b) Reciprocal Temperature (1000/K)

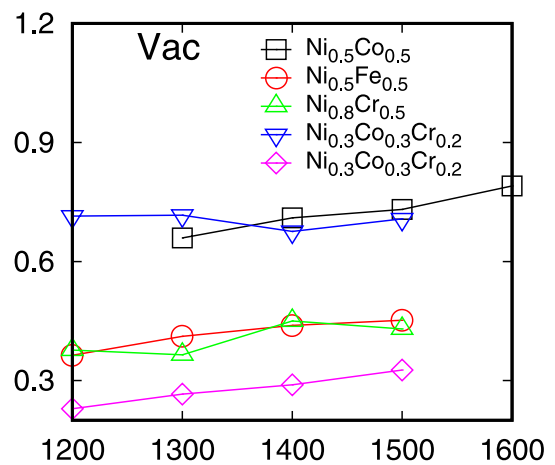

(d) Reciprocal Temperature (1000/K)

Figure 3: The ratio of partial diffusion coefficients $\left(D_{\mathrm{X}}^{*} / D_{\mathrm{Ni}^{\prime}}^{*}\right.$ where $\mathrm{X}$ denotes the other elements in the alloy) in considered alloys. In $\mathrm{Ni}_{0.4} \mathrm{Fe}_{0.4} \mathrm{Cr}_{0.2}$ $\left(\mathrm{Ni}_{0.333} \mathrm{Co}_{0.333} \mathrm{Cr}_{0.333}\right), D_{\mathrm{Fe}\left(\mathrm{C}_{0}\right)}^{*} / D_{\mathrm{Ni}}^{*}$ is presented by blue lines, whereas $D_{\mathrm{Cr}}^{*} / D_{\mathrm{Ni}}^{*}$ is denoted by pink lines. The results from the Bonny potential are provided in the left side, whereas those from the Zhou potential are given in the right.

dislocation line along the $[1 \overline{1} 2]$ axis and the Burgers vector along the [110] axis. Periodic boundary conditions were applied in both the [110] and [11̄2] directions. Fixed boundary conditions were used along the [111] direction. In this direction, the upper and lower regions of several atomic layers were fixed, whereas the atoms in the central part were mobile. The stress-controlled loading was applied by adding external forces to the atoms in the upper and lower regions. The magnitude of force was $F= \pm \sigma A / N_{ \pm}$, where $A$ is the area of [110]-[11 2$]$ plane, $N_{ \pm}$is the number of atoms in the upper or lower region respectively, and $\sigma$ is the stress applied to the dislocation. A time step of $1 \mathrm{fs}$ was used, and the total simulation time was around 1000 ps.

As reported previously [20, 22], for CSAs, steady dislocation movement can be observed only when the applied stress exceeds certain critical stress. Therefore, there are two modes for dislocation motion in CSAs: one is the obstacle-dominated motion at low stresses and the other is the smooth movement at high stresses [22]. The velocity of the dislocation can be fitted through the traveling distance versus time when the steady movement has achieved. The fitted results are displayed in Fig. 5.

Figure 5 indicates that the velocity of the dislocation first increases linearly with increasing applied stress, thus a slope can be defined from the velocity-stress curve. Previous studies suggest that defect accumulation in CSAs is well correlated with this slope [21]. Based on the Bonny potential, the slope of the considered CSAs is in the order of $\mathrm{Ni}_{0.8} \mathrm{Cr}_{0.2}>\mathrm{Ni}_{0.5} \mathrm{Fe}_{0.5}>$ $\mathrm{Ni}_{0.4} \mathrm{Fe}_{0.4} \mathrm{Cr}_{0.2}$. From the $\mathrm{Zhou}$ potential, the slope is in the order of $\mathrm{Ni}_{0.5} \mathrm{Co}_{0.5}>\mathrm{Ni}_{0.5} \mathrm{Fe}_{0.5} \approx \mathrm{Ni}_{0.8} \mathrm{Cr}_{0.2}>\mathrm{Ni}_{0.333} \mathrm{C}$ $\mathrm{o}_{0.333} \mathrm{Cr}_{0.333}$. These different features can be used to compare with their damage accumulation results. Another feature, the threshold stress required for steady dislocation movement also varies in different CSAs. For example, the Bonny potential indicates that $\mathrm{Ni}_{0.8} \mathrm{Cr}_{0.2}$ has a lower threshold stress than both $\mathrm{Ni}_{0.5} \mathrm{Fe}_{0.5}$ and $\mathrm{Ni}_{0.4} \mathrm{Fe}_{0.4} \mathrm{Cr}_{0.2}$. For the Zhou potential, it predicts that $\mathrm{Ni}_{0.8} \mathrm{Cr}_{0.2}$ and $\mathrm{Ni}_{0.333} \mathrm{Co}_{0.333} \mathrm{Cr}_{0.333}$ exhibit nearly the same threshold stress, higher than that in $\mathrm{Ni}_{0.5} \mathrm{Co}_{0.5}$ and $\mathrm{Ni}_{0.5} \mathrm{Fe}_{0.5}$.

\section{Damage accumulation}

Damage accumulation in different CSAs is simulated by accumulated 5-keV cascade simulations. A $34 \times 34 \times 34 \mathrm{fcc}$ supercell containing 157,216 atoms was used. The system was equilibrated first for $10 \mathrm{ps}$ at $300 \mathrm{~K}$ in an NPT ensemble. Then, a primary knocked-on atom (PKA) was chosen randomly in the box and given kinetic energy of $5 \mathrm{keV}$ to initiate the cascade. The simulation box was shifted so that the PKA was always located in the center of the box. A variable time step 
Bonny

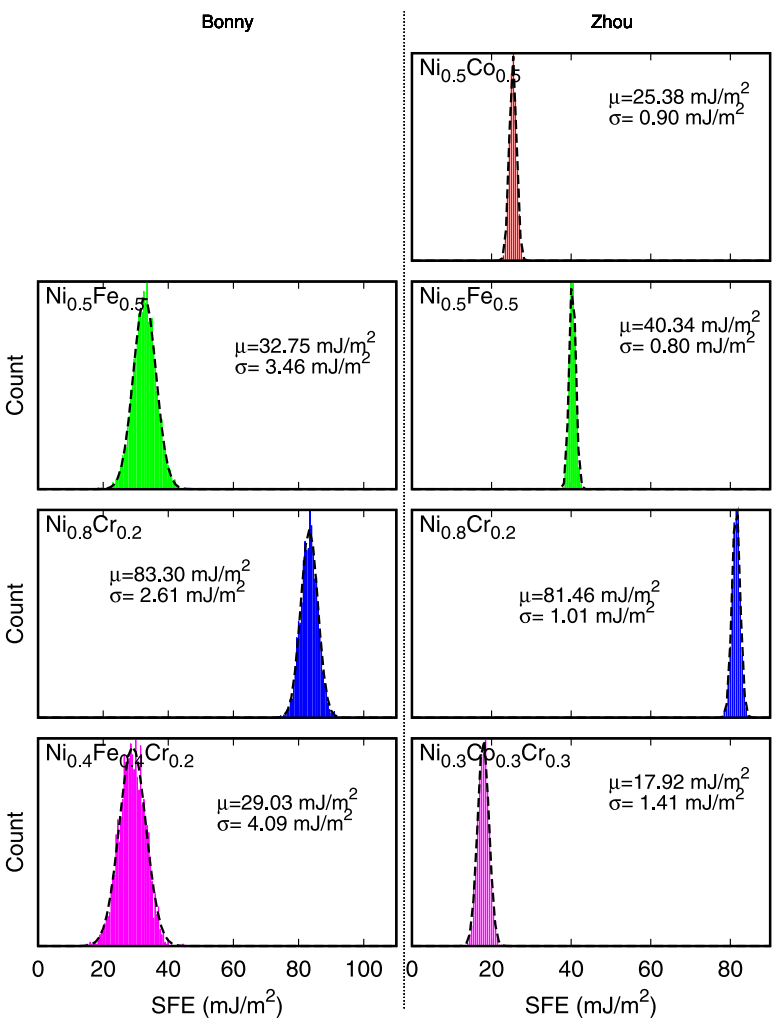

Figure 4: Distributions of stacking fault energies in considered alloys. The results from the Bonny potential are provided in the left column, whereas those from the Zhou potential are in the right.

scheme was adopted, in which the maximal displacement of all atoms was restricted within $0.01 \AA$. The total simulation time for the cascade was $30 \mathrm{ps}$, which was followed by a relaxation NPT simulation. A total of 600 cascades were simulated, which correspond to $0.25 \mathrm{dpa}$ (displacement per atom) based on the NRT standard [23]. The calculated defect number during the cascade simulation identified through the Wigner-Seitz defect analysis is given in Fig. 6. The interstitial number in large clusters $(N>51)$ is also calculated and shown in Fig. 7 .

The results from the Bonny potential suggest that the defect number in CSAs after 100 cascade simulations is remarkably lower than that generated in pure Ni. This result is consistent with previous simulations using the same potential [10]. On the other hand, the difference in the defect number from the Zhou potential is small in the considered alloys, which is also observed in previous studies [21]. Based on the Bonny potential, the defect evolution in $\mathrm{Ni}_{0.5} \mathrm{Fe}_{0.5}$ and $\mathrm{Ni}_{0.8} \mathrm{Cr}_{0.2}$ is similar, whereas $\mathrm{Ni}_{0.4} \mathrm{Fe}_{0.4} \mathrm{Cr}_{0.2}$ shows the lowest defect number after 600 cascade simulations. For the Zhou potential, $\mathrm{Ni}_{0.5} \mathrm{Fe}_{0.5}, \mathrm{Ni}_{0.8} \mathrm{Cr}_{0.2}$, and $\mathrm{Ni}_{0.333} \mathrm{Co}_{0.333} \mathrm{Cr}_{0.333}$ exhibit similar defect evolution after 500 cascade simulations, which is lower than that in pure $\mathrm{Ni}$ and $\mathrm{Ni}_{0.5} \mathrm{Co}_{0.5}$. Note that these results are averaged from three independent cascade simulations; it represents the defect evolution trend in these different alloys.
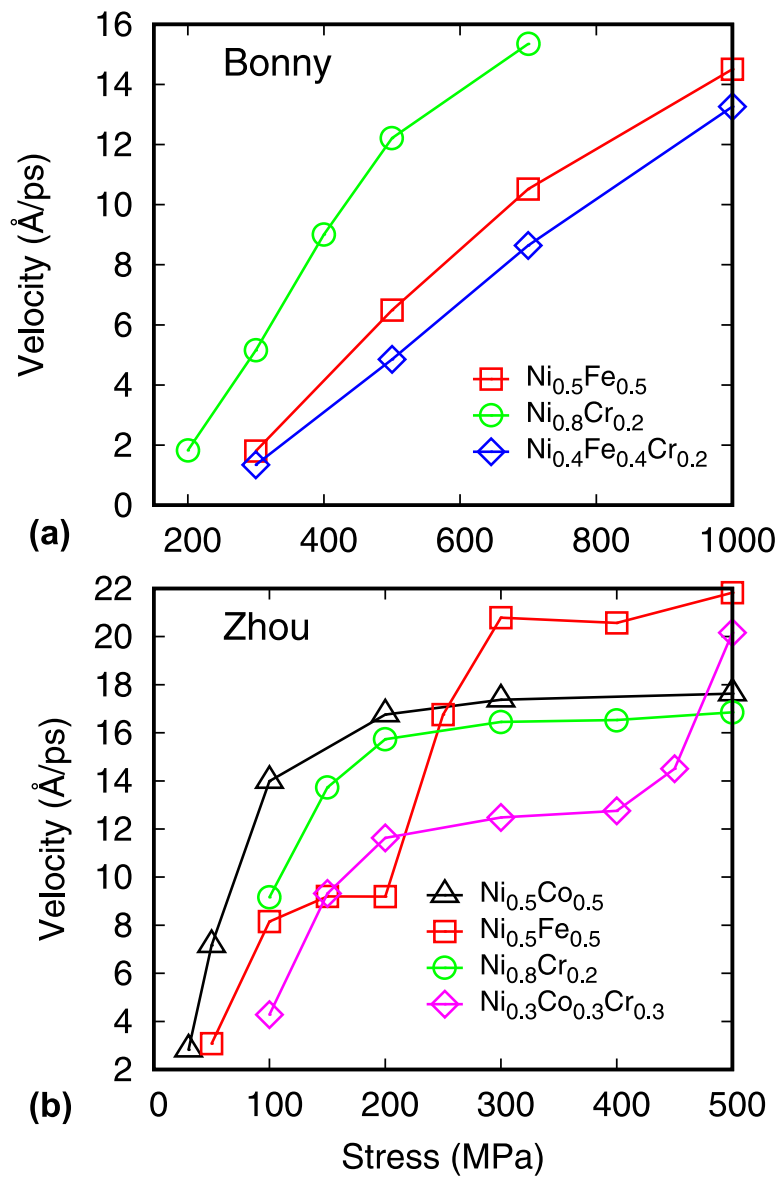

Figure 5: Velocities of an edge dislocation in considered alloys as a function of applied stress.

The interstitial number in large clusters indicates that ternary alloys exhibit the lowest number of interstitials for both potentials. The trend is consistent with the defect number induced by the accumulated cascade.

\section{Discussion}

Irradiation-induced changes in microstructure and mechanical properties of materials can be characterized by experimental techniques. Such observations are results of complexed defect creation, defect migration, and defect clustering. Yet, the contribution of these different processes can only be indirectly inferred from experiments. Atomistic simulations, on the other hand, can separate these different factors and provide details of the dominant contributors. For CSAs, experiments have demonstrated that their irradiation performance can be improved based on the structural heterogeneity, which depends on their compositions. Nevertheless, the details of heterogeneity remain elusive. By isolating different aspects of heterogeneity, this study provides a detailed analysis of the dominant roles influencing irradiation tolerance of CSAs. 


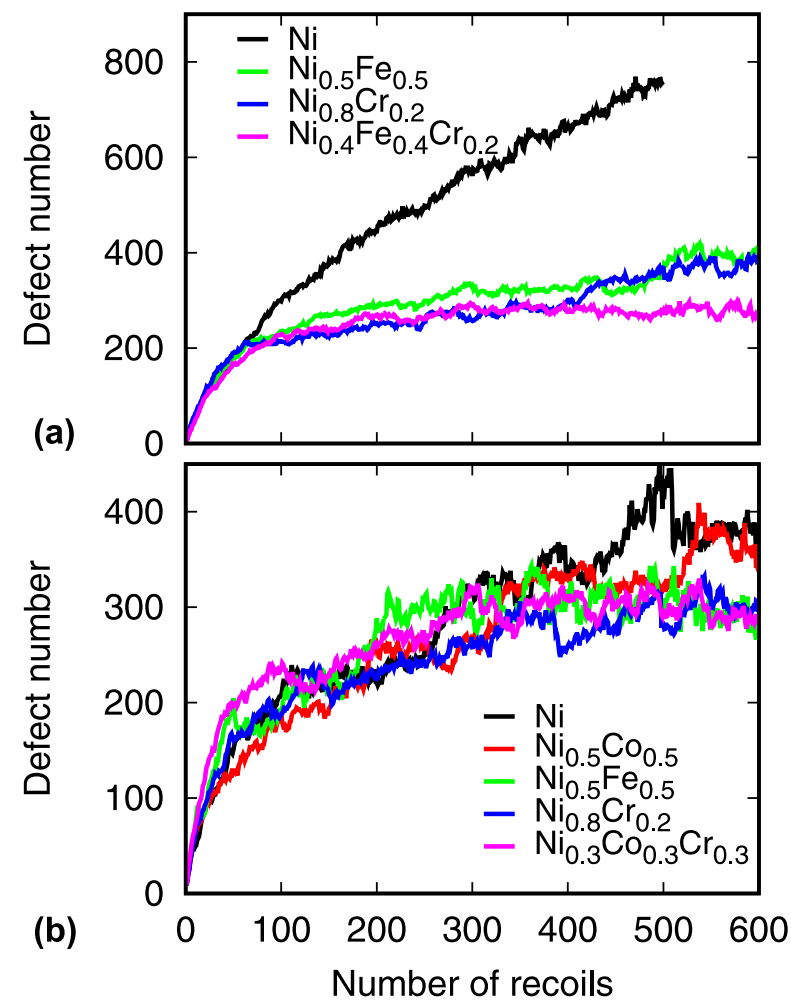

Figure 6: The defect number generated in the accumulated displacement cascade simulations. The upper row is the results from the Bonny potential, whereas the lower is the results from the Zhou potential.

The atomistic process is simulated by the MD method in this work. Although the MD method is inherently limited by its time $(\sim \mathrm{ns})$ and spatial $(\sim 10 \mathrm{~nm})$ scales, the defect accumulation feature in different CSAs is in good agreement with the available experiments $[8,9]$. For MD simulations, the most important factors determining the accuracy are the used empirical interatomic potentials. In this study, we have adopted two independently developed EAM potentials to minimize the uncertainty. In fact, these two potentials have both been used to simulate the irradiation response of different CSAs, and the obtained results are consistent with experiments [8, 21, 24].

The simulations were carried out in random solid-solution alloys, which correspond to single-phase solid-solution alloys that are used in most experiments, without any interfaces or grain boundaries. As such, the trend of defect number simulated is comparable to the damage accumulation observed in experiments. The results indicate similar defect evolution between $\mathrm{Ni}_{0.5} \mathrm{Fe}_{0.5}$ and $\mathrm{Ni}_{0.8} \mathrm{Cr}_{0.2}$, which is consistent with experimental results [25]. In addition, the lowest defect number in $\mathrm{Ni}_{0.4} \mathrm{Fe}_{0.4} \mathrm{Cr}_{0.2}$ and $\mathrm{Ni}_{0.333} \mathrm{Co}_{0.333} \mathrm{Cr}_{0.333}$ is also in accordance with the available experiments $[8,26]$. Thus, our simulations reproduce the damage evolution in the considered CSAs successfully. With this in mind, we can analyze the role of different aspects of heterogeneity played in defect evolution.

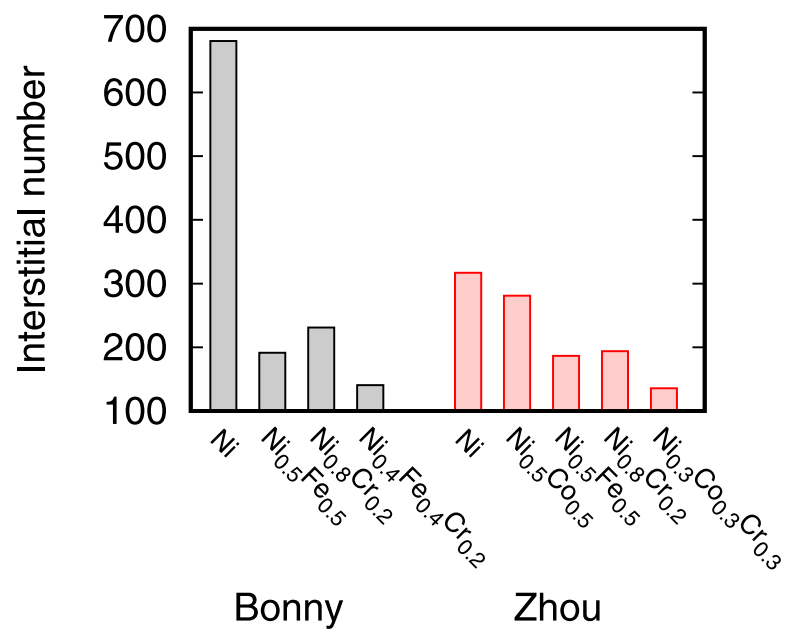

Figure 7: The number of interstitials in large defect clusters as obtained from the Bonny and Zhou potentials.

First, our results show that the lattice misfit does not correlate with defect evolution for all considered CSAs. For example, Bonny $\mathrm{Ni}_{0.8} \mathrm{Cr}_{0.2}$ exhibits the greatest lattice misfit, but the defect evolution in $\mathrm{Ni}_{0.8} \mathrm{Cr}_{0.2}$ is similar to $\mathrm{Ni}_{0.5} \mathrm{Fe}_{0.5}$. From the Zhou potential, $\mathrm{Ni}_{0.5} \mathrm{Co}_{0.5}$ possesses a rather small misfit, but the defect number in $\mathrm{Ni}_{0.5} \mathrm{Co}_{0.5}$ is not much different from that in $\mathrm{Ni}_{0.5} \mathrm{Fe}_{0.5}$ and $\mathrm{Ni}_{0.8} \mathrm{Cr}_{0.2}$. Second, the correlation with mixing energy is also invalid. The Bonny $\mathrm{Ni}_{0.5} \mathrm{Fe}_{0.5}$ shows the most negative mixing energy, yet the defect number in $\mathrm{Ni}_{0.5} \mathrm{Fe}_{0.5}$ is actually higher than that in $\mathrm{Ni}_{0.4} \mathrm{Fe}_{0.4} \mathrm{Cr}_{0.2}$ with rather higher mixing energy. The Zhou potential leads to rather different mixing energies for $\mathrm{Ni}_{0.5} \mathrm{Fe}_{0.5}$, $\mathrm{Ni}_{0.8} \mathrm{Cr}_{0.2}$, and $\mathrm{Ni}_{0.333} \mathrm{Co}_{0.333} \mathrm{Cr}_{0.333}$, yet the defect evolution in these three alloys is similar. These results suggest that structural properties, such as lattice misfit and enthalpy of mixing, are not correlated with specific defect evolution in CSAs, and we need to look into the defect properties.

Compared to those structural parameters, atomic-level defect properties seem to have a larger impact on defect evolution for CSAs. In particular, the wide distribution of migration energies of interstitials and vacancies correlates well with the defect number observed in accumulated cascade. That is to say, the larger fluctuations in the migration energy distributions, the lower defect number is observed. The distribution is the manifest of defect energy landscape peculiar in CSAs. A wider distribution suggests a rougher defect energy landscape, which leads to heterogeneous defect diffusion that can contribute efficiently to defect recombination. Therefore, characterizing defect energy distributions is an important measure to probe the irradiation resistance of CSAs. Besides the point defect properties, the distribution of SFEs also relates to defect evolution because it governs dislocation behavior. In particular, a larger fluctuation magnitude in SFEs tends to 
make dislocation motion more difficult, as seen by the threshold stress required for steady dislocation motion displayed in Fig. 5. The dislocation motion becomes more difficult, which contributes to the lower defect number observed in accumulated cascade.

The defect diffusion at finite temperature represents average effects from migration energy distributions. Compared with the diffusion coefficients provided in Fig. 2, we do not find a correlation between diffusion coefficients and defect evolution. Nevertheless, the preferential diffusion of defects indeed shows such correlations. From Fig. 3, it shows $\mathrm{Ni}_{0.4} \mathrm{Fe}_{0.4} \mathrm{Cr}_{0.2}$ possesses the highest $D_{\mathrm{Cr}}^{*} / D_{\mathrm{Ni}}^{*}$ and the lowest $D_{\mathrm{Fe}}^{*} / D_{\mathrm{Ni}}^{*}$ ratio for both the interstitial and vacancy diffusion based on the Bonny potential. For the Zhou potential, $D_{\mathrm{Co}}^{*} / D_{\mathrm{Ni}}^{*}$ is the highest and $D_{\mathrm{Cr}}^{*} / D_{\mathrm{Ni}}^{*}$ is the lowest in $\mathrm{Ni}_{0.333} \mathrm{Co}_{0.333} \mathrm{Cr}_{0.333}$. The large chemical anisotropy in defect diffusion correlates with the low defect number in accumulated cascade. In this case, diffusion is mainly mediated by one or two components in the alloy, and the defect accumulation is suppressed due to the chemically biased diffusion-enhanced defect recombination [27].

\section{Conclusion}

The detailed roles of atomic-level heterogeneity in influencing irradiation performance of CSAs are examined by atomistic simulations in this work. Our results show that structural parameters for CSAs, such as lattice misfit and mixing energy, have little influence on defect accumulation in CSAs. Instead, we demonstrate that atomic-level defect properties, such as the distribution of defect migration energies, preferential defect diffusion, and the distribution of SFEs, have a close correlation with the defect number created by cascades. Thus, this work highlights defect properties as a reliable figure of merit for irradiation resistance in CSAs. Note that the irradiation resistance studied here is represented by the defect number under cascades, which is one of the important criteria to measure the irradiation response of materials. This work provides basic information on the governing factors for irradiation performance of CSAs, which is helpful for future studies aiming to develop irradiation-resistant concentrated alloys.

\section{Method}

All simulations were performed using the open-source largescale atomic/molecular massively parallel simulator (LAMMPS) [28]. The interatomic interactions were described using the EAM potentials parameterized by Bonny et al. [14] and Zhou et al. [15]. In the Zhou potential, we have included the parameterization for $\mathrm{Cr}$ from Lin et al. [29]. However, the original version of the $\mathrm{Cr}$ potential leads to instabilities in alloys, and slight modification was made based on the analysis of Anand et al. [30]. These potentials have been successfully used to model damage evolution in corresponding alloys previously $[8,10,21,24,25]$.

\section{Acknowledgments}

This work was supported by City University of Hong Kong (No. 9610425), Research Grants Council of Hong Kong (No. 21200919), and National Natural Science Foundation of China (No. 11975193).

\section{References}

1. D.B. Miracle and O.N. Senkov: A critical review of high entropy alloys and related concepts. Acta Mater. 122, 448-511 (2017).

2. B. Cantor, I.T.H. Chang, P. Knight, and A.J.B. Vincent: Microstructural development in equiatomic multicomponent alloys. Mater. Sci. Eng., A 375, 213-218 (2004).

3. J-W. Yeh, S-K. Chen, S-J. Lin, J-Y. Gan, T-S. Chin, T-T. Shun, C-H. Tsau, and S-Y. Chang: Nanostructured high-entropy alloys with multiple principal elements: Novel alloy design concepts and outcomes. Adv. Eng. Mater. 6, 299-303 (2004).

4. Y. Zhang, S. Zhao, W.J. Weber, K. Nordlund, F. Granberg, F. Djurabekova, F. Granbergc, and F. Djurabekova: Atomic-level heterogeneity and defect dynamics in concentrated solid-solution alloys. Curr. Opin. Solid State Mater. Sci. 21, 221-237 (2017).

5. C. Varvenne, A. Luque, and W.A. Curtin: Theory of strengthening in fcc high entropy alloys. Acta Mater. 118, 164-176 (2016).

6. Y.N. Osetsky, L.K. Béland, A.V. Barashev, and Y. Zhang: On the existence and origin of sluggish diffusion in chemically disordered concentrated alloys. Curr. Opin. Solid State Mater. Sci. 22, 65-74 (2018).

7. S. Zhao, Y. Osetsky, and Y. Zhang: Preferential diffusion in concentrated solid solution alloys: $\mathrm{NiFe}, \mathrm{NiCo}$, and $\mathrm{NiCoCr}$. Acta Mater. 128, 391-399 (2017).

8. F. Granberg, K. Nordlund, M.W. Ullah, K. Jin, C. Lu, H. Bei, L.M. Wang, F. Djurabekova, W.J. Weber, and Y. Zhang: Mechanism of radiation damage reduction in equiatomic multicomponent single phase alloys. Phys. Rev. Lett. 116, 135504 (2016).

9. Y. Zhang, G.M. Stocks, K. Jin, C. Lu, H. Bei, B.C. Sales, L. Wang, L.K. Béland, R.E. Stoller, G.D. Samolyuk, M. Caro,

A. Caro, and W.J. Weber: Influence of chemical disorder on energy dissipation and defect evolution in concentrated solid solution alloys. Nat. Commun. 6, 8736 (2015).

10. M. Jin, P. Cao, and M.P. Short: Thermodynamic mixing energy and heterogeneous diffusion uncover the mechanisms of radiation damage reduction in single-phase Ni-Fe alloys. Acta Mater. 147, 16-23 (2018).

11. S. Zhao, T. Egami, G.M. Stocks, and Y. Zhang: Effect of $d$ electrons on defect properties in equiatomic $\mathrm{NiCoCr}$ and $\mathrm{NiCoFeCr}$ concentrated solid solution alloys. Phys. Rev. Mater. 2 , 013602 (2018) 
12. S. Zhao, W.J. Weber, and Y. Zhang: Unique challenges for modeling defect dynamics in concentrated solid-solution alloys. JOM 69, 2084-2091 (2017).

13. A. Barashev, Y. Osetsky, H. Bei, C. Lu, L. Wang, and Y. Zhang: Chemically-biased diffusion and segregation impedes void growth in irradiated Ni-Fe alloys. Curr. Opin. Solid State Mater. Sci. 23, 92-100 (2019).

14. G. Bonny, N. Castin, and D. Terentyev: Interatomic potential for studying ageing under irradiation in stainless steels: The $\mathrm{FeNiCr}$ model alloy. Modell. Simul. Mater. Sci. Eng. 21, 85004 (2013).

15. X.W. Zhou, R.A. Johnson, and H.N.G. Wadley: Misfit-energyincreasing dislocations in vapor-deposited $\mathrm{CoFe} / \mathrm{NiFe}$ multilayers. Phys. Rev. B 69, 144113 (2004).

16. C. Varvenne, A. Luque, W.G. Nöhring, and W.A. Curtin: Average-atom interatomic potential for random alloys. Phys. Rev. B 93, 104201 (2016).

17. G. Henkelman, B.P. Uberuaga, and H. Jónsson: A climbing image nudged elastic band method for finding saddle points and minimum energy paths. J. Chem. Phys. 113, 9901-9904 (2000).

18. Y.N. Osetsky, L.K. Béland, and R.E. Stoller: Specific features of defect and mass transport in concentrated fcc alloys. Acta Mater. 115, 364-371 (2016)

19. S. Zhao, Y. Osetsky, G.M. Stocks, and Y. Zhang: Localenvironment dependence of stacking fault energies in concentrated solid-solution alloys. npj Comput. Mater. 5, 13 (2019).

20. S. Zhao, Y.N. Osetsky, and Y. Zhang: Atomic-scale dynamics of edge dislocations in $\mathrm{Ni}$ and concentrated solid solution NiFe alloys. J. Alloys Compd. 701, 1003-1008 (2017).
21. E. Levo, F. Granberg, C. Fridlund, K. Nordlund, and

F. Djurabekova: Radiation damage buildup and dislocation evolution in $\mathrm{Ni}$ and equiatomic multicomponent Ni-based alloys. $J$. Nucl. Mater. 490, 323-332 (2017).

22. Y.N. Osetsky, G.M. Pharr, and J.R. Morris: Two modes of screw dislocation glide in fcc single-phase concentrated alloys. Acta Mater. 164, 741-748 (2019).

23. M. Norgett, M. Robinson, and I. Torrens: A proposed method of calculating displacement dose rates. Nucl. Eng. Des. 33, 50 (1975).

24. M.W. Ullah, D.S. Aidhy, Y. Zhang, and W.J. Weber: Damage accumulation in ion-irradiated Ni-based concentrated solidsolution alloys. Acta Mater. 109, 17-22 (2016).

25. M.W. Ullah, H. Xue, G. Velisa, K. Jin, H. Bei, W.J. Weber, and Y. Zhang: Effects of chemical alternation on damage accumulation in concentrated solid-solution alloys. Sci. Rep. 7, 4146 (2017).

26. G. Velişa, M.W. Ullah, H. Xue, K. Jin, M.L. Crespillo, H. Bei, W.J. Weber, and Y. Zhang: Irradiation-induced damage evolution in concentrated Ni-based alloys. Acta Mater. 135, 54-60 (2017).

27. S. Zhao, Y. Osetsky, A.V. Barashev, and Y. Zhang: Frenkel defect recombination in $\mathrm{Ni}$ and $\mathrm{Ni}$-containing concentrated solidsolution alloys. Acta Mater. 173, 184-194 (2019).

28. S. Plimpton: Fast parallel algorithms for short-range molecular dynamics. J. Comput. Phys. 117, 1-19 (1995).

29. Z. Lin, R.A. Johnson, and L.V. Zhigilei: Computational study of the generation of crystal defects in a bcc metal target irradiated by short laser pulses. Phys. Rev. B 77, 214108 (2008).

30. G. Anand, R. Goodall, and C.L. Freeman: Role of configurational entropy in body-centred cubic or face-centred cubic phase formation in high entropy alloys. Scr. Mater. 124, 90-94 (2016). 